\title{
Integrated Path-tracking and Control Allocation Controller for Autonomous Electric Vehicle under Limit Handling Condition
}

\author{
Boyuan Li, Javad Ahmadi, Chenhui Lin, Efstathios Siampis, Stefano Longo, Senior Member, IEEE \\ and Efstathios Velenis
}

\begin{abstract}
In current literature, a number of studies have separately considered path-tracking (PT) control and control allocation (CA) method, but few of studies have integrated them together. This study proposes an integrated PT and CA method for autonomous electric vehicle with independent steering and driving actuators in the limit handling scenario. The high-level feedback PT controller can determine the desired total tire forces and yaw moment, and is designed to guarantee yaw angle error and lateral deviation converge to zero simultaneously. The low-level CA method is formulated as a compact quadratic programming (QP) optimization formulation to optimally allocate individual control actuator. This CA method is designed for a prototype experiment electric vehicle with particularly steering and driving actuator arrangement. The proposed integrated PT controller is validate through numerical simulation based on a high-fidelity CarMaker model on highspeed limit handling scenario.
\end{abstract}

\section{INTRODUCTION}

Safety in vehicular traffics can be the most celebrated issue in the context of on-road vehicle driving. However, the uncertainties caused by human drivers may cause serious safety issues for on-road vehicle. Autonomous vehicle driving is an attractive research area to tackle the safety issue caused by uncertainties of human driver. Path following control as an important part of an autonomous vehicle driving is an active research area recently [1-4]. In [1], a path-tracking (PT) control strategy based on Autodriver algorithm for frontwheel-steering autonomous vehicles has been developed and a control loop is introduced to compensate the present errors generated by the differences of the desired location on the road and the actual position of the vehicle. Another lateral control strategy of an autonomous vehicle using integrated backstepping and sliding mode controller is reported in [2]. In addition, a nonsingular terminal sliding mode and active disturbance rejection controller are used for path following control of autonomous ground vehicle [3].

On the other hand, control allocation (CA) of redundant actuators with the aim of increased maneuverability and fault tolerance of the system have been employed in many dynamic systems including vehicular applications [5-8]. For example, Bagheri et al. integrate adaptive sliding mode control and unscented Kalman filter estimator to improve vehicle yaw

* Research supported by Innovate UK through AID-CAD project

B. Li, J. Ahmadi, C. Lin, E. Siampis, S. Longo and E. Velenis are with the Centre for Automotive Engineering, School of Aerospace, Transport and Manufacturing, Cranfield University, Cranfield MK43 0AL, U.K. (e-mail: stability by differential braking [5]. In [6], a direct yaw moment controller based on adaptive sliding mode method is designed for an electric vehicle with four in-wheel motors. Furthermore, an adaptive vehicle lateral-plane motion control using optimal tire friction forces with saturation limits consideration is reported [7].

While the above studies separately consider PT and CA problems, the PT control performance can be greatly improved when CA method is integrated together. Wang et al. argue that the CA method for independent driving vehicle can enhance safety and handling by utilizing actuator redundancies, particularly in severe driving conditions, and a better PT performance can be achieved for the integration method [8]. Hu et al. also suggest that for the PT control, CA method can offer flexible actuation with rapid torque response, which is likely to lend the strong support for tracking control issues [9]. Furthermore, a comparative study of different PT and CA methods has been carried out in [10]. The comparison simulation results suggest that including the CA method in PT controller can improve vehicle control performance compared with PT controller only based on the steering actuator. This study also compares the results between the separate PT and CA structure (where PT only sends the reference steering angle to $\mathrm{CA}$ ) and integrated structure (where PT sends both reference steering angle and control yaw moment to CA). Compared with separate structure, the integrated structure controller can enhance the agility of the vehicle and is suitable for racing car in limit handling scenario.

Our study proposes an integrated structure controller consisting of high-level PT controller and low-level CA method for the autonomous electric vehicle with independent steering and driving actuators under the limit handling condition. The high-level PT feedback controller, which can determine the desired total tire forces and yaw moment, is designed based on a nonlinear path-following vehicle dynamics model which including the yaw angle error and lateral deviation error. The feedback control strategy is designed to guarantee yaw angle error and lateral deviation converge to zero simultaneously and the stability of the feedback controller has been proved. The desired velocity is determined by optimal velocity profile which can be precalculated offline. The desired values of generalized forces
boyuan.li@cranfield.ac.uk;Javad.Ahmadi@cranfield.ac.uk;chenhui.lin@cra nfield.ac.uk;Efstathios.Siampis@cranfield.ac.uk;s.longo@cranfield.ac.uk;e. velenis@cranfield.ac.uk).

J. Ahmadi is also with the Department of Mechanical Engineering, Payame Noor University, P.O. Box 19395-3697, Tehran, Iran. 
and moment in high-level controller are mapped to the physical control actuators by optimal CA strategy in the lower level. This low-level CA method, which is formulated as a compact quadratic programming (QP) optimization formulation, is designed particularly for a prototype electric vehicle which has one driving motor on the front axle and two independent driving motors installed on rear wheels. This prototype vehicle also has front and rear wheel independent steering characteristic. In order to improve the computational efficiency, the time-efficient solver generated by Forces Pro is applied to solve optimization problem of CA in this study, which can satisfy the requirement of real-time PT control [11]. The proposed integrated PT controller is validate through numerical simulation based on a high fidelity CarMaker model on high-speed limit handling scenario.

This rest of the paper is organized as follows. In section II, the dynamic modeling and the proposed control system is presented. Section III describes simulation results to evaluate the developed controller. Summary and conclusion are given in section IV.

\section{VEHICLE DYNAMICS MODELING AND CONTROLLER DESIGN}

The whole integrated controller includes the high-level PT controller and low-level CA controller. According to desired path and desired velocity profile, the high-level PT controller can determine the desired total longitudinal tire force, the desired total lateral tire force and desired yaw moment. In order to achieve desired tire forces and yaw moment, the lowlevel CA optimally allocates individual steering and driving actuators.

\section{A. High-level PT controller}

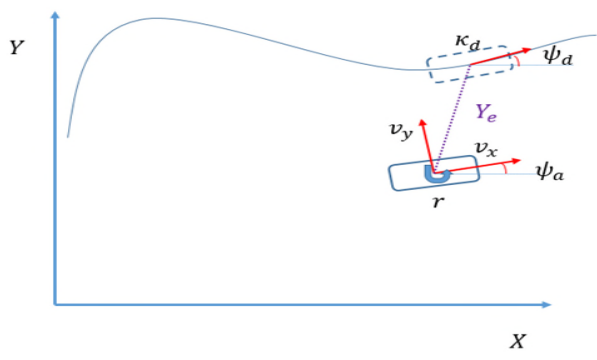

Figure 1. Path following vehicle dynamics model

The high-level controller is designed based on the following nonlinear vehicle dynamics model [8], which is illustrated in Figure 1:

$$
\begin{gathered}
\dot{v}_{x}=v_{y} r+\frac{F_{x d}}{m} \\
\dot{v}_{y}=-v_{x} r+\frac{F_{y d}}{m} \\
\dot{r}=\frac{M_{z d}}{I_{z}} \\
\dot{Y}_{e}=v_{x} \sin \psi_{e}+v_{y} \cos \psi_{e} \\
\dot{\psi}_{e}=r-\kappa_{d} v_{x}
\end{gathered}
$$

where $v_{x}, v_{y}, r$ are vehicle longitudinal velocity, lateral velocity and yaw rate. $F_{x d}, F_{y d}, M_{z d}$ represent total longitudinal tire force, total lateral tire force and yaw moment. $\psi_{e}$ is the yaw angle tracking error and $Y_{e}$ is the lateral position tracking error. $\psi_{e}=\psi_{a}-\psi_{d} . m$ and $I_{z}$ are the vehicle mass and moment of inertial in terms of yaw axis. $\kappa_{d}$ is the curvature of the desired path.

In this study, the desired forward velocity $v_{x d}$ is determined by a set velocity $v_{\text {set }}$ and an optimal velocity profile $v_{m}$ which can be pre-calculated offline. This optimal velocity profile can make the autonomous vehicle travel along the desired path with maximum acceleration and minimum time [12]. If $v_{\text {set }}<v_{m}$, then $v_{x d}=v_{\text {set }}$, otherwise $v_{x d}=$ $v_{m}$.

In order to achieve the desired forward speed, the following equivalent total tire force control input in the longitudinal direction of vehicle is calculated:

$$
F_{x d}=m\left(-r v_{y}+\dot{v}_{x d}-k_{1} e_{1}\right)
$$

where $e_{1}=v_{x}-v_{x d}$. For $k_{1}>0$, the above control law leads to the following stable error dynamics and the stability of longitudinal vehicle dynamics can be proved according to 1(a):

$$
\dot{e}_{1}+k_{1} e_{1}=0
$$

On the other hand, in order to achieve path following, yaw angle tracking error $\psi_{e}$ and lateral position tracking error $Y_{e}$ should converge to zero simultaneously. For regulation of $Y_{e}$, equation (1d) is rewritten as the following equation:

$$
\ddot{Y}_{e}=\dot{v}_{x} \sin \psi_{e}+\dot{v}_{y} \cos \psi_{e}+\dot{\psi}_{e}\left(v_{x} \cos \psi_{e}-v_{y} \sin \psi_{e}\right)
$$

According to (1b), equation (4) can be written as:

$$
\ddot{Y}_{e}=\frac{F_{y d}}{m} \cos \psi_{e}+B+C
$$

where $B=\dot{v}_{x} \sin \psi_{e}+\dot{\psi}_{e}\left(v_{x} \cos \psi_{e}-v_{y} \sin \psi_{e}\right)$,

$C=-v_{x} r \cos \psi_{e}$.

In order to regular $\psi_{e}$ simultaneously with $Y_{e}, B$ can be rewritten as following equation according to (1e):

$$
B=\dot{v}_{x} \sin \psi_{e}+\left(r-\kappa_{d} v_{x}\right)\left(v_{x} \cos \psi_{e}-v_{y} \sin \psi_{e}\right)
$$

Therefore, the equivalent total lateral control force is calculated as following equation according to (1d):

$$
F_{y d}=\frac{m}{\cos \psi_{e}}\left[-B-C-k_{2}\left(v_{x} \sin \psi_{e}+v_{y} \cos \psi_{e}\right)-k_{3} Y_{e}\right]
$$

If $k_{2}>0$ and $k_{3}>0$ are satisfied, applying the above control law (7) into (5) leads to the following stable error dynamics equation:

$$
\ddot{Y}_{e}+k_{2} \dot{Y}_{e}+k_{3} Y_{e}=0
$$


In order to determine the desired controlled yaw moment, equation (1c) can be rewritten as following equation:

$$
M_{z d}=\dot{r} I_{z}=\left(\ddot{\psi}_{e}+\dot{r}_{d}\right) I_{z}
$$

The equivalent yaw moment is determined as following according to (9) and (1e):

$$
M_{z d}=\left[-k_{4}\left(r-\kappa_{d} v_{x}\right)-k_{5} \psi_{e}+\dot{r}_{d}\right] I_{z}
$$

If $k_{4}>0$ and $k_{5}>0$ are satisfied, applying the above control law (10) into (1c) leads to the following stable error dynamics equation:

$$
\ddot{\psi}_{e}+k_{4} \dot{\psi}_{e}+k_{5} \psi_{e}=0
$$

In summary, the total desired longitudinal force, lateral force and yaw moment can be determined by (2), (7) and (10). In order to ensure the stability in steady-state, desired longitudinal acceleration $\dot{v}_{x d}$ and desired yaw acceleration $\dot{r}_{d}$ can be assumed as zero.

\section{B. Low-level CA method}

In this section, low-level CA is presented to allocate individual actuator to achieve the total desired tire forces and yaw moment in high-level. The low-level CA is designed based on a special prototype electric vehicle test platform. This test vehicle is designed by Delta Motorsports for the AIDCAV project, which has three driving motors with one motor on the front axle and two independent driving motors on the rear-left wheel and rear-right wheel, respectively. This vehicle also has front-and-rear-wheel independent steering characteristic. The following CA model is proposed based on this vehicle structure [13]:

$$
\begin{gathered}
\sum_{i=1,2,3} u_{i}=F_{x d} \\
C_{\alpha f}\left(2 u_{4}-\alpha_{f l d}-\alpha_{f r d}\right)+C_{\alpha r}\left(2 u_{5}+\alpha_{r l d}+\alpha_{r r d}\right)=F_{y d} \\
(12 \mathrm{~b}) \\
-\frac{1}{2} b_{r} u_{2}+\frac{1}{2} b_{r} u_{3}+l_{f} C_{\alpha f}\left(2 u_{4}-\alpha_{f l d}-\alpha_{f r d}\right)- \\
l_{r} C_{\alpha f}\left(2 u_{5}+\alpha_{r l d}+\alpha_{r r d}\right)=M_{z d}
\end{gathered}
$$

where allocated control actuator inputs includes longitudinal tire force on front axle and two rear wheels $u_{1}=\frac{T_{t f}}{R_{\omega}}, u_{2}=$ $\frac{T_{t r l}}{R_{\omega}}, u_{3}=\frac{T_{t r r}}{R_{\omega}}$, and front and rear wheel steering angles $u_{4}=$ $\delta_{f}$ and $u_{5}=\delta_{r} . T_{t f}, T_{t r l}, T_{r r}$ are motor torque of front axle, rear left wheel and rear right wheel, respectively. $R_{\omega}$ is the wheel radius. $l_{f}$ and $l_{r}$ are the front and rear wheel base lengths, while $b_{f}$ and $b_{r}$ are the front and rear track widths. $C_{\alpha f}$ and $C_{\alpha r}$ are cornering stiffness of front tire and rear tire, respectively. $\alpha_{f l d}, \alpha_{f r d}, \alpha_{r l d}, \alpha_{r r d}$ are side-slip angle of individual wheel [14]:

$$
\begin{aligned}
& \alpha_{f l d}=\tan ^{-1}\left(\frac{v_{y d}+l_{f} r}{v_{x d}-0.5 b_{f} r}\right) \\
& \alpha_{f r d}=\tan ^{-1}\left(\frac{v_{y d}+l_{f} r}{v_{x d}+0.5 b_{f} r}\right)
\end{aligned}
$$

$$
\begin{aligned}
& \alpha_{r l d}=\tan ^{-1}\left(\frac{l_{r} r_{d}-v_{y d}}{v_{x d}-0.5 b_{r} r}\right) \\
& \alpha_{r r d}=\tan ^{-1}\left(\frac{l_{r} r_{d}-v_{y d}}{v_{x d}+0.5 b_{r} r}\right)
\end{aligned}
$$

It is assumed $v_{y d}=0$.

Based on (12)(13), the optimization cost function of CA problem can be formulated as a compact QP formulation:

$$
\begin{aligned}
& \min _{u_{i, i=1,3,5}} J=\left(\boldsymbol{\tau}_{\boldsymbol{R} H \boldsymbol{S}}-B \boldsymbol{u}\right)^{\prime} Q_{1}\left(\boldsymbol{\tau}_{\boldsymbol{R} H \boldsymbol{S}}-B \boldsymbol{u}\right)+\boldsymbol{u}^{\prime} Q_{2} \boldsymbol{u} \\
& \approx \boldsymbol{u}^{\prime}\left(B^{\prime} Q_{1} B+Q_{2}\right) \boldsymbol{u}+\left(-2 B Q_{1} \boldsymbol{\tau}_{\boldsymbol{R} H S}\right) \boldsymbol{u}
\end{aligned}
$$

where $\boldsymbol{u}^{\prime}=\left[\begin{array}{lllll}u_{1} & u_{2} & u_{3} & u_{4} & u_{5}\end{array}\right]$,

$$
\begin{gathered}
B=\left[\begin{array}{ccccc}
1 & 1 & 1 & 0 & 0 \\
0 & 0 & 0 & 2 C_{\alpha f} & 2 C_{\alpha r} \\
0 & -0.5 b_{r} & 0.5 b_{r} & 2 l_{f} C_{\alpha f} & 2 l_{r} C_{\alpha r}
\end{array}\right], \\
\boldsymbol{\tau}_{\boldsymbol{R H S}}=\left[\begin{array}{c}
F_{y d}+C_{\alpha f}\left(\alpha_{f l d}+\alpha_{f r d}\right)-C_{\alpha r}\left(\alpha_{r l d}+\alpha_{r r d}\right) \\
M_{z d}+l_{f} C_{\alpha f}\left(\alpha_{f l d}+\alpha_{f r d}\right)+l_{r} C_{\alpha r}\left(\alpha_{r l d}+\alpha_{r r d}\right)
\end{array}\right],
\end{gathered}
$$

$Q_{1}$ is a 3-by-3 diagonal matrix which presents the weighting factors of control targets $F_{x d}, F_{y d}$ and $M_{x z d} \cdot Q_{2}$ is a $5-$ by-5 diagonal matrix which determines the penalty weighting factors of individual actuator. The optimisation problem (14) should also satisfy friction circle constraint and physical limit of steering and driving actuators [13], which is not detailed explained here.

In order to show the advantage of CA optimization formulation (14), two alternative formulations of CA are presented and the simulation results will be compared in section III. First, the optimisation cost function of CA when torque-vectoring is disabled can be formulated as:

$$
\min _{u_{i, i=1,2,3,4} J} \approx \boldsymbol{u}^{\prime}\left(B^{\prime} Q_{1} B+Q_{2}\right) \boldsymbol{u}+\left(-2 B Q_{1} \boldsymbol{\tau}_{\boldsymbol{R} H \boldsymbol{S}}\right) \boldsymbol{u}
$$

where $\boldsymbol{u}^{\prime}=\left[\begin{array}{llll}u_{1} & u_{2} & u_{3} & u_{4}\end{array}\right], u_{1}=\frac{T_{t f}}{R_{\omega}}, u_{2}=\frac{2 T_{t r l}}{R_{\omega}}=\frac{2 T_{t r r}}{R_{\omega}}$, $u_{3}=\delta_{f}, u_{4}=\delta_{r}, B=\left[\begin{array}{cccc}1 & 1 & 0 & 0 \\ 0 & 0 & 2 C_{\alpha f} & 2 C_{\alpha r} \\ 0 & 0 & 2 l_{f} C_{\alpha f} & 2 l_{r} C_{\alpha r}\end{array}\right]$.

Secondly, the optimisation cost function of CA when rearwheel steering is disabled can be formulated as:

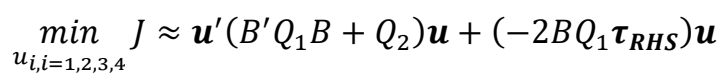

where $\boldsymbol{u}^{\prime}=\left[\begin{array}{llll}u_{1} & u_{2} & u_{3} & u_{4}\end{array}\right], u_{1}=\frac{T_{t f}}{R_{\omega}}, u_{2}=\frac{T_{t r l}}{R_{\omega}}, u_{3}=$ $\frac{T_{t r r}}{R_{\omega}}, u_{4}=\delta_{f}, B=\left[\begin{array}{cccc}1 & 1 & 1 & 0 \\ 0 & 0 & 0 & 2 C_{\alpha f} \\ 0 & -0.5 b_{r} & 0.5 b_{r} & 2 l_{f} C_{\alpha f}\end{array}\right] . Q_{2}$ in optimisation problems (15)(16) is reduced as a 4-by-4 matrix to reflect the penalty scaling factors of individual actuator.

In order to improve the computational efficiency and satisfy the needs of real-time CA optimisation, the above CA optimisation problem with QP formulation can be solved by 
time-efficient solver based on primal dual interior point (PDIP) method [15] in Forces Pro.

It is noted that the high-level PT controller and low-level CA method are not separately considered but are integrated together through the total desired forces and yaw moment in this study. The tuning of PT control gains $k_{1-5}$ and weighing factor matrix $Q_{1}, Q_{2}$ in CA method can both greatly affect the overall system stability and control performance, so all these control gains and scaling factors should be carefully chosen to achieve good performance.

\section{Simulation ReSUlts}

In this section, simulation results based on a high-fidelity vehicle model from CarMaker software is presented to validate the performance of proposed controller. The CarMaker vehicle model provided by Delta Motorsports can accurately present the vehicle dynamics performance of experimental test vehicle of AID-CAV project. The main parameters of CarMaker model are shown in Table I. The simulation is carried out on a small challenge section of Silverstone track with two sharp turns to evaluate the limit handling performance of controller. The desired path for tracking is the centerline of the track and the desired velocity is determined by the set velocity and velocity profile. In order to show the advantage of proposed integrated PT and CA method, the simulation results of CA method when torque-vectoring is disabled and simulation results of CA method when rear-wheel steering is disabled are presented and compared. The CA method when torquevectoring is disabled is simply represented as 'CA without TV' and the CA method when rear-wheel steering is disabled is simply represented as 'CA without RS'.

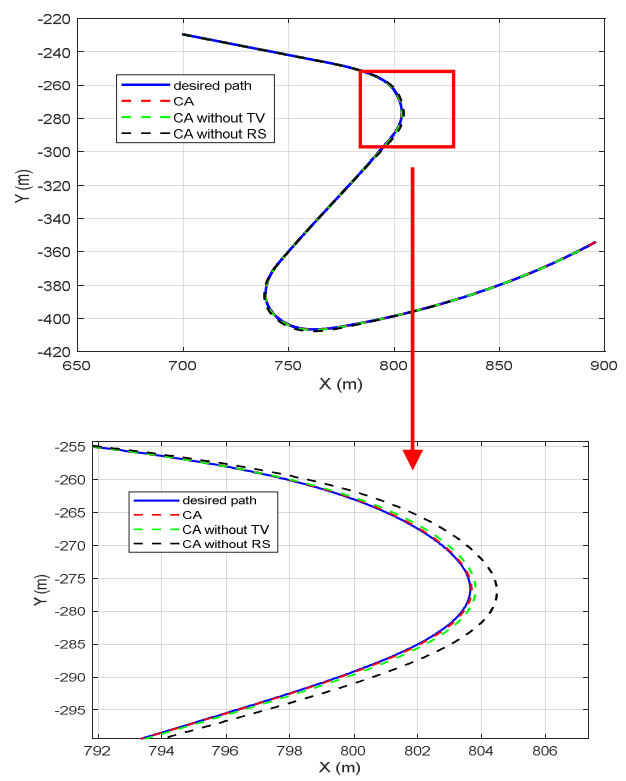

Figure 2. The PT performance $\left(v_{\text {set }}=80 \mathrm{~km} / \mathrm{h}\right)$

TABLE I

MAIN PARAMETERS OF CARMAKER MODEL

\begin{tabular}{lll}
\hline \hline $\begin{array}{c}\text { Symbo } \\
1\end{array}$ & Quantity & Values \\
\hline$m$ & Vehicle total mass & $700.28 \mathrm{~kg}$
\end{tabular}

\begin{tabular}{lcc}
$I_{z}$ & Moment inertial & $1597.717 \mathrm{~kg} \cdot \mathrm{m}^{2}$ \\
& around yaw axle & \\
$l_{f}$ & Front wheel base & $0.999 \mathrm{~m}$ \\
$l_{r}$ & Rear wheel base & $0.996 \mathrm{~m}$ \\
$b_{f}$ & Front track width & $1.52 \mathrm{~m}$ \\
$b_{r}$ & Rear track width & $1.52 \mathrm{~m}$ \\
$R_{\omega}$ & Wheel radius & $0.32 \mathrm{~m}$ \\
$C_{\alpha}$ & Cornering stiffness & $29220 \mathrm{~N} / \mathrm{rad}$ \\
$\mu$ & Tire-road friction & 1 \\
\hline \hline
\end{tabular}

TABLE II

SENSOR NOISE PARAMETERS

\begin{tabular}{lcc}
\hline \hline \multicolumn{1}{c}{ Vehicle state } & White noise level & $\begin{array}{c}\text { White noise } \\
\text { frequency }(\mathrm{Hz})\end{array}$ \\
\hline Longitudinal velocity & $5 \times 10^{-6}$ & 1000 \\
Body slip angle & $5 \times 10^{-6}$ & 1000 \\
Longitudinal acceleration & 0.03 & 1000 \\
Lateral acceleration & 0.06 & 1000 \\
Yaw rate & $3 \times 10^{-4}$ & 1000 \\
\hline \hline
\end{tabular}

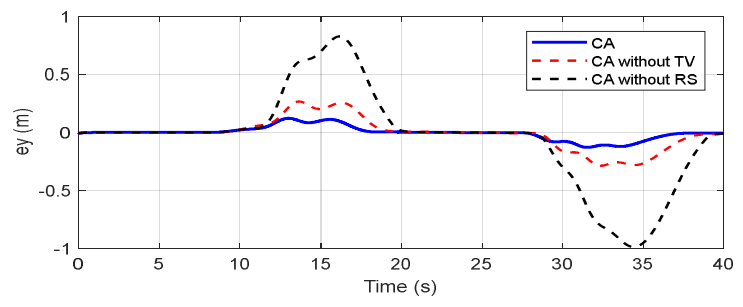

(a)

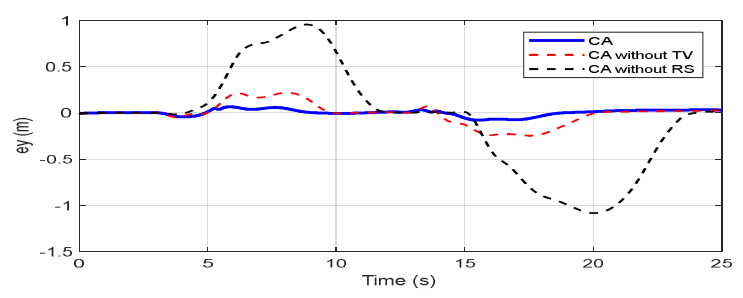

(b)

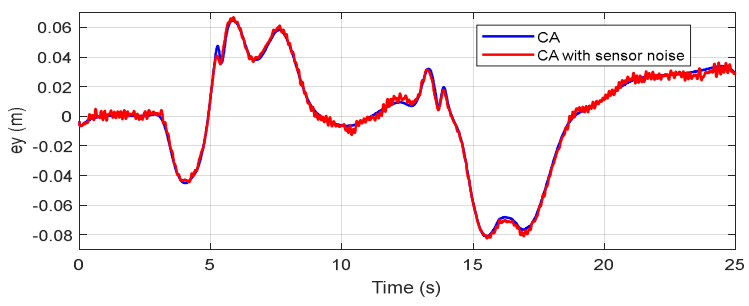

(c)

Figure 3. The lateral PT error (a) $v_{\text {set }}=30 \mathrm{~km} / \mathrm{h}$; (b) $v_{\text {set }}=80 \mathrm{~km} / \mathrm{h}$ (c) $v_{\text {set }}=80 \mathrm{~km} / \mathrm{h}$ with sensor noise

Figure 2 and Figure 3 show the PT performance when the set velocity is $30 \mathrm{~km} / \mathrm{h}$ and $80 \mathrm{~km} / \mathrm{h}$. According to Figure 3 , the PT performance of CA method can accurately track the desired path and shows obviously advantageous over CA without TV and CA without RS. Figure 3(c) analyses the sensitivity of proposed controller to the sensor noise (the detailed parameters of white noise are shown in Table II), and suggests the robustness performance. Figure 4(a) suggests the 
actual velocity is close to $30 \mathrm{~km} / \mathrm{h}$ when the set velocity is 30 $\mathrm{km} / \mathrm{h}$. In this case, the desired velocity is the same as the set velocity. Figure 4(b) shows that although the set velocity is 80 $\mathrm{km} / \mathrm{h}$, the actual velocity drops to only $40 \mathrm{~km} / \mathrm{h}$ at $5 \mathrm{~s}$ and $15 \mathrm{~s}$ when the autonomous vehicle is making the sharp turning. In this case, the desired velocity is determined together by set velocity and velocity profile. If the set velocity is smaller than velocity profile, the desired velocity is set velocity, otherwise the set velocity is higher than the velocity profile (such as $5 \mathrm{~s}$ and $15 \mathrm{~s}$ in Figure 4(b)) and the desired velocity is the velocity profile. It is noted that in order to guarantee the vehicle stability, the speed limit for CA and CA without TV is set as $77 \%$ of the velocity profile, while the CA without RS is set as $60 \%$ of the velocity profile. Thus, the velocity of CA without RS during sharp turning is lower than other methods in Figure 4(b).

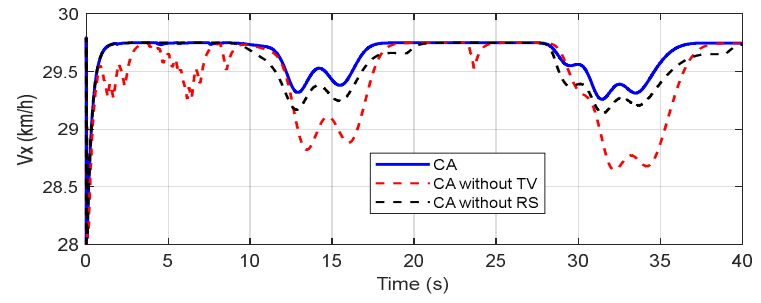

(a)

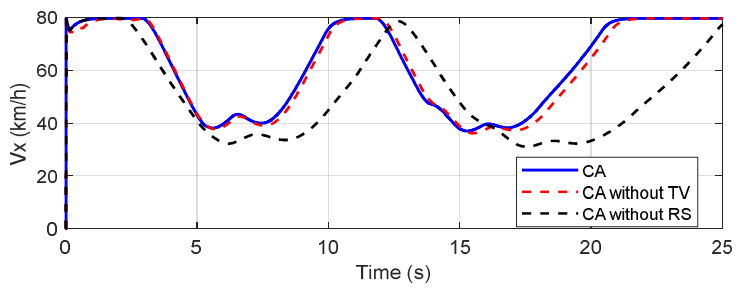

(b)

Figure 4. Actual vehicle longitudinal velocity (a) $v_{\text {set }}=$ $30 \mathrm{~km} / \mathrm{h}$; (b) $v_{\text {set }}=80 \mathrm{~km} / \mathrm{h}$

Figure 5 shows the normalized acceleration of vehicle when the set velocity is $30 \mathrm{~km} / \mathrm{h}$ and $80 \mathrm{~km} / \mathrm{h}$, which can be calculated by the following equation:

$$
\bar{a}=\frac{\sqrt{a_{x}^{2}+a_{y}^{2}}}{\mu g}
$$

where $a_{x}$ and $a_{y}$ are vehicle longitudinal and lateral acceleration. Figure 5 suggests that the normalized acceleration is quite small when set velocity is $30 \mathrm{~km} / \mathrm{h}$, but the normalized acceleration increases to over 0.8 (close to friction limit) when the set velocity is $80 \mathrm{~km} / \mathrm{h}$. Figure 5 (c) shows the effect of the sensor noise on the acceleration response of proposed method.

Figure 6 and Figure 7 present the allocated individual motor torque and individual wheel steering angle when the set velocity is $80 \mathrm{~km} / \mathrm{h}$. The motor torque and steering angle response when set velocity is $30 \mathrm{~km} / \mathrm{h}$ is not presented here due to the limit of the space. Figure 6(a) shows that for CA method, driving motors in real left wheel and rear right wheel has torque-vectoring performance during the sharp-turning at 4 s, while Figure 6(b) shows for CA without TV method, the motor torques of rear left wheel and rear right wheel are same. Figure 6(c) suggests that for CA without RS method, the two rear motors have more obvious torque-vectoring performance to achieve the PT since rear-wheel steering is disabled. According to Figures 7(a)(b), CA method without TV requires larger steering angle compared with $\mathrm{CA}$ method since the torque-vectoring of rear driving motors are disabled. Figure 7(c) suggests that rear wheel steering angle is zero for the CA without RS method.

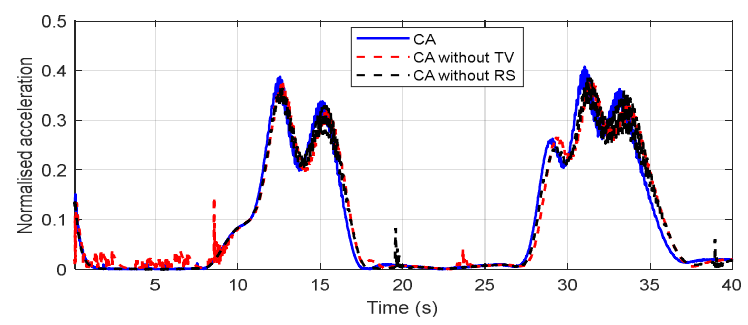

(a)

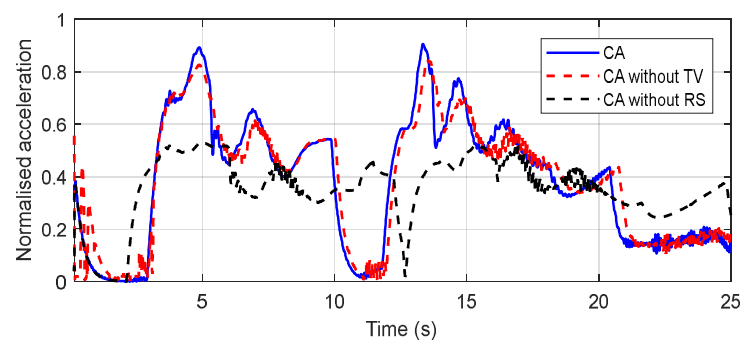

(b)

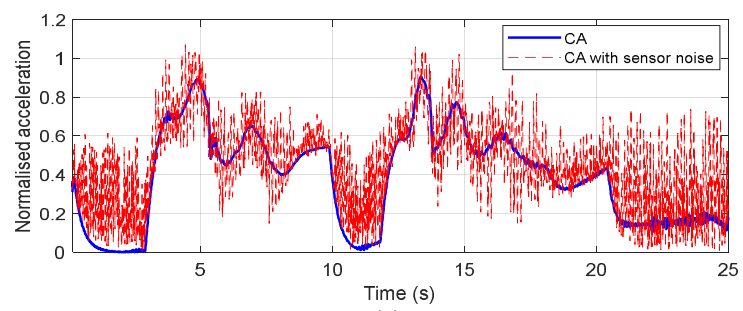

(c)

Figure 5. Actual normalized acceleration (a) $v_{\text {set }}=$ $30 \mathrm{~km} / \mathrm{h}$; (b) $v_{\text {set }}=80 \mathrm{~km} / \mathrm{h}$; (c) $v_{\text {set }}=80 \mathrm{~km} / \mathrm{h}$ with sensor noise

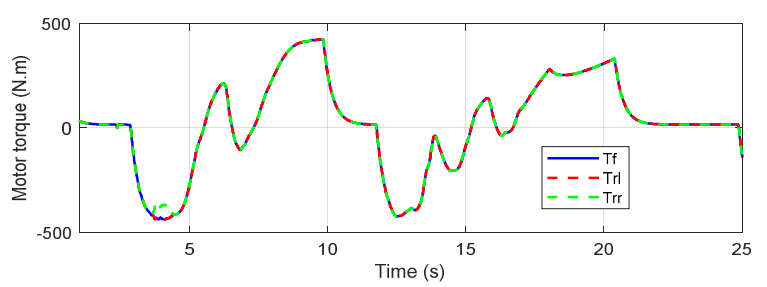

(a)

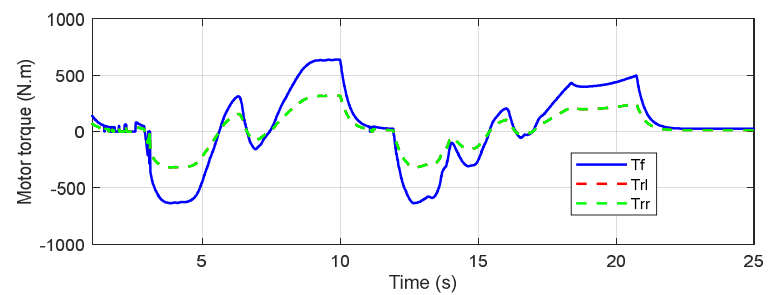


(b)

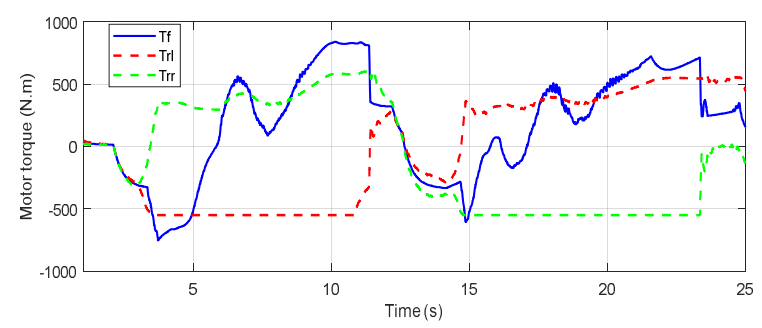

(c)

Figure 6. Actual motor torque (a) CA; (b) CA without TV; (c) CA without RS.

It is noted only simulation results of single section of a specific track is presented in this section due to the page limit, and more results will be included in the future extended journal paper.

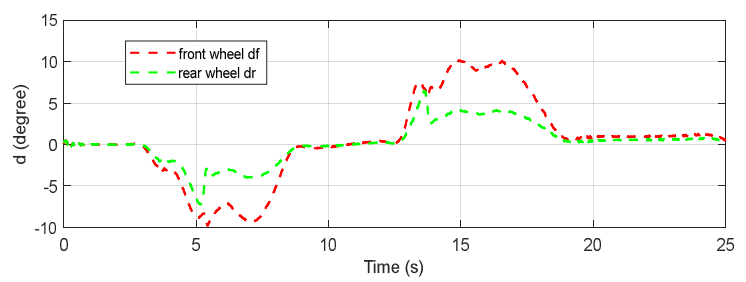

(a)

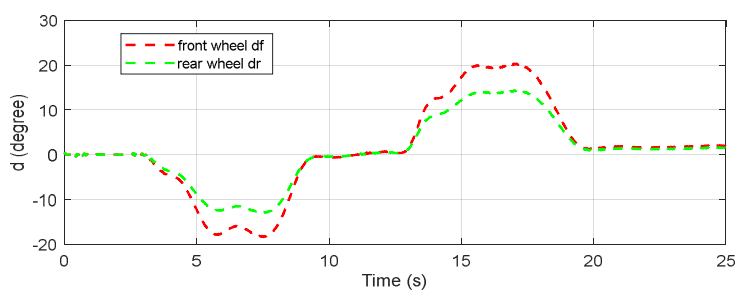

(b)

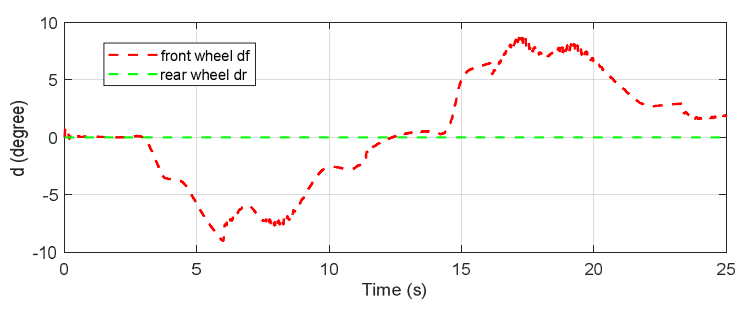

(c)

Figure 7. Actual wheel steering angle (a) CA; (b) CA without $\mathrm{TV}$; (c) CA without RS.

\section{CONCLUSION}

This study proposes an integrated PT and CA method for autonomous electric vehicle in the high-speed limit handling scenario. The simulation results base on a high-fidelity CarMaker model proves that the proposed integrated controller can accurately tracking the desired path with lateral tracking error smaller than $0.2 \mathrm{~m}$ in high-speed turning scenario. The proposed low-level CA method shows better path following performance compared with the results when the torque-vectoring of $\mathrm{CA}$ is disabled and when the rearwheel steering of CA is disabled. In the future, the proposed integrated controller will be tested on the experiment test platform provided by Delta Motorsports, and the real-time path following performance can be validated.

\section{REFERENCES}

[1] H. Marzbani, H. Khayyam, C. N. TO and R. N. Jazar, "Autonomous Vehicles- Autodriver Algorithm and Vehicle Dynamics," IEEE Trans. Veh. Technol., vol. 68, no. 4, pp. 6379-6390, 2019.

[2] A. Norouzi, M. Masoumi, A. Barari and S. F. Sani, "Lateral control of an autonomous vehicle using integrated backstepping and sliding mode controller," Proceedings of the Institution of Mechanical Engineers, Part K: Journal of Multi-body Dynamics, vol. 233, no. 1, pp. 141-151, 2019.

[3] Y. Wu, L. Wang, J. Zhang, and F. Li, "Path Following Control of Autonomous Ground Vehicle Based on Nonsingular Terminal Sliding Mode and Active Disturbance Rejection Control," IEEE Trans. Veh. Technol., vol. 68, no. 7, pp. 6379-6390, 2019.

[4] Y. Wu , L. Wang, J. Zhang, and F. Li, "Should the Desired Heading in Path Following of Autonomous Vehicles be the Tangent Direction of the Desired Path," IEEE Trans. Veh. Technol., vol. 16, no. 6, pp. 6379-6390, 2015.

[5] A. Bagheri, S. Azadi, A. Soltani, "A combined use of adaptive sliding mode control and unscented Kalman filter estimator to improve vehicle yaw stability," Proceedings of the Institution of Mechanical Engineers, Part K: Journal of Multi-body Dynamics, vol. 231, no.2, pp. 388-401, 2016.

[6] A. N. Asiabar and R. Kazemi, "A direct yaw moment controller for a four in-wheel motor drive electric vehicle using adaptive sliding mode control," Proceedings of the Institution of Mechanical Engineers, Part K: Journal of Multi-body Dynamics, vol. 233, no.3, pp. 549-567, 2019.

[7] J. Ahmadi, A. K. Sedigh, "Adaptive vehicle lateral-plane motion control using optimal tire friction forces with saturation limits consideration," IEEE Transactions on Vehicular Technology, vol. 58, no. 8, pp. 4098-4107, 2009.

[8] R. Wang, C. Hu, F. Yan, and M. Chadli, "Composite nonlinear feedback control for path following of four-wheel independently actuated autonomous ground vehicles," IEEE Trans. Intell. Transp. Syst., vol. 17, no. 7, pp. 2063-2074, Mar. 2016.

[9] C. Hu, R. Wang, F. Yan, and N. Chen, "Output constraint control on path following of four-wheel independently actuated autonomous ground vehicles," IEEE Trans. Veh. Technol., vol. 65, no. 6, pp. 4033-4043, 2016.

[10] C. Chatzikomis, A. Sorniotti, P. Gruber, M. Zanchetta, D. Willans, and B. Balcombe, "Comparison of Path Tracking and TorqueVectoring Controllers for Autonomous Electric Vehicles", IEEE Transactions on Intelligent Vehicles, vol.3, no.4, pp.559-570, 2018.

[11] A. Domahidi and J. Jerez, "FORCES Professional," embotech GmbH, Zürich, Switzerland, Tech. Rep., Jul. 2014. [Online]. Available: http:// embotech.com/FORCES-Pro

[12] E. Velenis and P. Tsiotras, "Minimum-Time Travel for a Vehicle with Acceleration Limits: Theoretical Analysis and Receding-Horizon Implementation", Journal of Optimization Theory and Applications, vol.138, pp.275-296, 2008.

[13] B. Li, E. Siampis, C. Lin, S. Longo and E. Velenis, "A Time-efficient Integrated Path-tracking and Control Allocation Method for Autonomous Electric Vehicle," 2019 IEEE 58th Conference on Decision and Control (CDC), Nice, France, 2019, pp. 6700-6705.

[14] B. L. Boada, M. J.L. Boada and V. Díaz, "Fuzzy-logic applied to yaw moment control for vehicle stability", Vehicle System Dynamics, vol.43, no.10, pp.753-770, 2005.

[15] A. Domahidi, A. Zgraggen, M.N. Zeilinger, M. Morari, C.N. Jones, "Efficient interior point methods for multistage problems arising in receding horizon control", Conference on Decision and Control (CDC), Maui, HI, USA, December 2012, pp.668-674. 
2021-01-08

\section{Integrated Path-tracking and Control}

\section{Allocation Controller for Autonomous}

\section{Electric Vehicle under Limit Handling Condition}

\section{$\mathrm{Li}$, Boyuan}

IEEE

Li B, Lin C, Siampis E, et al., (2020) Integrated Path-tracking and Control Allocation Controller for Autonomous Electric Vehicle under Limit Handling Condition. In: 2020 IEEE Intelligent Vehicles Symposium (IV), 19 October - 13 November 2020, Las Vegas, NV

https://doi.org/10.1109/IV47402.2020.9304608

Downloaded from Cranfield Library Services E-Repository 